\title{
DOS NUEVAS ESPECIES DE ENCARSIA FOERSTER (HYMENOPTERA: APHELINIDAE) DE LOS ESTADOS DE SAN LUIS POTOSÍ Y TABASCO, MÉXICO
}

\section{SVETLANA N. MYARTSEVA, ENRIQUE RUIZ-CANCINO* \& JUANA MARÍA CORONADO-BLANCO}

Museo de Insectos, Facultad de Ingeniería y Ciencias, Universidad Autónoma de Tamaulipas, 87149

Cd. Victoria, Tamaulipas, México.<smyartse@uat.edu.mx>; <jmcoronado@uat.edu.mx>

*Autor corresponsal: <eruiz@uat.edu.mx>

Myartseva, S. N., E. Ruiz-Cancino \& J. M. Coronado-Blanco. 2012. Dos nuevas especies de Encarsia Foerster (Hymenoptera: Aphelinidae) de los estados de San Luis Potosí y Tabasco, México. Acta Zoológica Mexicana (n. s.), 28(2): 457-464.

RESUMEN. Se describen dos especies nuevas del género Encarsia Foerster (Aphelinidae), E. xilitla sp. nov. y E. yumka sp. nov., colectadas en los estados de San Luis Potosí y Tabasco, México.

Palabras clave: Himenópteros, avispas parasitoides, nuevas especies.

Myartseva, S. N., E. Ruiz-Cancino \& J. M. Coronado-Blanco. 2012. Two new species of Encarsia Foerster (Hymenoptera: Aphelinidae) from the states of San Luis Potosi and Tabasco, Mexico. Acta Zoológica Mexicana (n. s.), 28(2): 457-464.

ABSTRACT. Two new species of the genus Encarsia Foerster (Aphelinidae), E. xilitla sp. nov. and E. yumka sp. nov., collected in the states of San Luis Potosi and Tabasco, Mexico, are described.

Key words: Hymenopterans, parasitoid wasps, new species.

\section{INTRODUCCIÓN}

Aphelinidae es una familia de avispas parasitoides de la superfamilia Chalcidoidea y del orden Hymenoptera. El género Encarsia Foerster es uno de los más diversos en esta familia, con 344 especies descritas (Heraty et al. 2007). En México ocurren 88 especies de Encarsia (Myartseva \& Evans 2008).

Estas avispas calcidoideas se comportan como parasitoides primarios (raramente secundarios) principalmente de ninfas de mosquitas blancas (familia Aleyrodidae), escamas armadas (familia Diaspididae) y de escamas suaves (familia Coccidae), pero también parasitan pulgones hormafidinos (fam. Aphididae), todos del orden Hemiptera. Algunas especies son parasitoides de huevos de diversos insectos (Polaszek 1991).

Recibido: 04/01/2012; aceptado: 09/05/2012. 
Varias especies de Encarsia tienen un rol importante en el control biológico de hemípteros plaga en todo el mundo, predominantemente de mosquitas blancas y de escamas armadas. A pesar de su gran importancia, la fauna, taxonomía y biología de las especies de Encarsia han sido insuficientemente estudiadas en México. En los últimos años, los autores han encontrado y descrito nuevas especies de este género en varios estados del país (Myartseva et al. 2008, 2009, 2010, 2011; Myartseva 2007, 2009; Myartseva \& González-Hernández 2008). El objetivo de este artículo fue describir dos especies nuevas del género Encarsia, colectadas en localidades de San Luis Potosí y de Tabasco, México.

\section{MATERIAL Y MÉTODOS}

Se colectaron muestras de plantas infestadas con mosquitas blancas en Xilitla, San Luis Potosí, (98 59' de longitud oeste y $21^{\circ}$ 23’ de latitud norte) y en Yumká, Tabasco ( $92^{\circ} 58^{\prime}$ de longitud oeste y $17^{\circ} 59^{\prime}$ de latitud norte) siguiendo el método propuesto por Noyes (1982). El material fue llevado al Laboratorio de Control Biológico de la Facultad de Ingeniería y Ciencias (FIC) de la Universidad Autónoma de Tamaulipas (UAT), donde se prepararon laminillas en bálsamo de Canadá. Para estudiar detalles de su morfología (cabeza, mesosoma y gáster) se utilizó un microscopio binocular Leica MZ6 y un microscopio compuesto Leica CME.

Para la identificación del género y de las especies de Encarsia se utilizaron varias claves: la clave de géneros de Chalcidoidea neárticos (Woolley 1997), las claves de especies de Myartseva y Evans (2008), Hayat (1998), Schmidt y Polaszek (2007), De Santis (1948), Huang y Polaszek (1998) y las de Nikolskaya y Yasnosh (1966). Además, se estudiaron las descripciones originales de algunas especies para comparar con las especies nuevas de Encarsia. El material se encuentra depositado en el Entomological Research Museum, University of California-Riverside (ERM-UCR) y en el Museo de Insectos de la Facultad de Ingeniería y Ciencias, Universidad Autónoma de Tamaulipas (MIFA).

\section{RESULTADOS \\ Encarsia xilitla Myartseva, sp. nov.}

Coloración. Cabeza amarilla, frontovértice anaranjado oscuro con una mancha parduzca cerca de los ocelos, occipucio con una mancha oval oscura alrededor del foramen, margen superior de la boca negro a los lados del clípeo, espacio malar negro, clípeo parduzco. Antenas pardas, radícula amarillenta, escapo pardo claro. Mandíbula amarillo oscuro. Palpos labiales y maxilares blancuzcos. Ojos negros, ocelos rojizos. Pronoto negro. Lóbulo medio del mesoescudo amarillo con una mancha triangular oscura medialmente, axilas pardas, lóbulos laterales amarillos con una mancha ahumada apicalmente, escutelo amarillo con una mancha oscura alrededor de la base 
de cada seta escutelar posterior. Setas del mesoescudo negras. Alas anteriores hialinas, venación ahumada. Patas amarillo blancuzco, fémures posteriores ligeramente ahumados en el margen dorsal, segmento apical de los tarsos ligeramente ahumado. Pecíolo pardo oscuro, con el margen posterior claro. Gáster negro parduzco, último terguito amarillo un poco oscurecido, tercera válvula blancuzca, puntas de los estiletes negros.

Estructura. Cabeza tan ancha como el mesosoma, ligeramente más ancha que alta. Frontovértice cerca de 0.6 veces la anchura de la cabeza. Distancia del ocelo posterior al ojo cerca de 0.7 veces de la distancia entre los ocelos posteriores. Ojos setosos, cerca de 1.3 veces tan largos como las mejillas. Antenas (Fig. 1) insertadas cerca del margen bucal. Distancia entre los tórulos 0.8 veces tan larga como la distancia del tórulo al ojo. Radícula antenal 2.5 veces tan larga como ancha. Escapo cerca de 4.9 veces tan largo como ancho. Pedicelo 1.5 veces tan largo como ancho. El primer segmento funicular es el más corto, cerca de 2 veces tan largo como ancho, segundo y tercer segmentos subiguales en longitud, cada uno cerca de 2.5 veces tan largo como ancho. Maza antenal de 3 segmentos, no más ancha y ligeramente más larga que el funículo. Segmentos flagelares 2-6 cada uno con una sensila larga y delgada. Lóbulo medio del mesoescudo con 18-20 setas; cada axial con una seta, cada lóbulo lateral con 3 setas. Escutelo 0.4 veces tan largo como el lóbulo medio y 2 veces tan ancho como largo. Sensilas escutelares placoideas ampliamente espaciadas, separadas por una distancia aproximada de 6 diámetros de una sensila. Par anterior de setas escutelares 0.8 veces tan largo como el par posterior. Distancia entre las setas anteriores cerca de 0.9 veces tan larga como la distancia entre las posteriores. Ala anterior uniformemente setosa, cerca de 2.4 veces tan larga como ancha, su fleco marginal cerca de 0.2 veces la anchura alar, base con 9-10 setas cortas situadas en arco. Vena marginal (Fig. 2) más o menos tan larga como la vena submarginal y con 9-10 setas largas a lo largo del margen anterior. Ala posterior cerca de 7 veces tan larga como ancha, su fleco marginal visiblemente más largo que la anchura del ala. Fórmula tarsal 5-45. Espuela de la tibia media 0.8 veces tan larga como el basitarso (Fig. 3). Terguitos gástricos 2-7 con 2, 2, 2, 4, 6 y 6 setas, respectivamente. Ovipositor (Fig. 4) muy poco expuesto, originado en el nivel del terguito 3 , sólo 1.1 veces más largo que la tibia media, tercera válvula cerca de 0.6 veces tan larga como el segundo valvífer.

Longitud del cuerpo: $0.9-1.0 \mathrm{~mm}$.

\section{Macho desconocido.}

Comentarios. Según Myartseva y Evans (2008), Encarsia xilitla sp. nov. pertenece al grupo de especies luteola. La nueva especie es cercana a E. catemaco Myartseva E. guadeloupae Viggiani, de las cuales se distingue por: E. catemaco presenta cabeza negra, lóbulo medio del mesoescudo negro, escutelo completamente amarillo claro, tercera válvula parduzca, maza antenal de dos segmentos, primer segmento flagelar con sensila y el ovipositor 1.8 veces tan largo como la tibia media. Por su parte, E. xilitla 


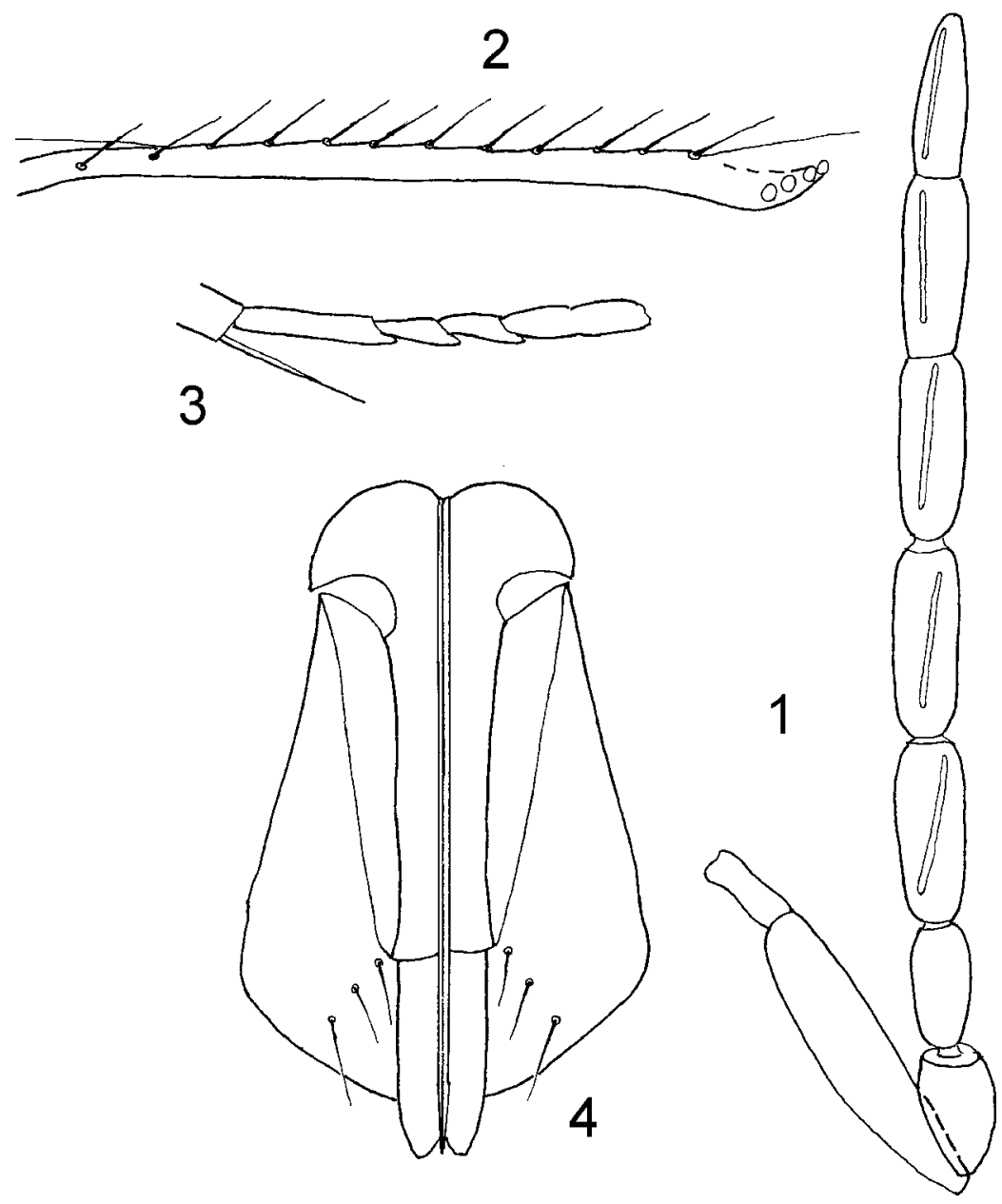

Figuras 1-4. Encarsia xilitla Myartseva sp. nov., hembra: 1 - antena, 2 - vena marginal del ala anterior, 3 - tarso de la pata media, 4 - ovipositor.

tiene la cabeza amarilla con el frontovértice anaranjado oscuro y una mancha parduzca cerca de los ocelos, lóbulo medio del mesoescudo amarillo con una mancha triangular oscura, escutelo con una mancha oscura alrededor de la base de cada seta escutelar posterior, tercera válvula blancuzca, maza antenal de tres segmentos, primer segmento flagelar sin sensila y ovipositor sólo 1.1 veces más largo que la tibia media.

E. guadeloupae tiene la cabeza pardo oscuro, escutelo completamente amarillo, ala anterior con 4 grupos de setas basales, vena marginal con 6-7 setas; terguito gástrico 7 con 4 setas y la tercera válvula 0.7 veces tan larga como el segundo valvífer. $E$. 
xilitla tiene la cabeza amarilla con el frontovértice anaranjado oscuro y una mancha parduzca cerca de los ocelos, lóbulo medio del mesoescudo amarillo con una mancha triangular oscura medialmente, escutelo con una mancha oscura alrededor de la base de cada seta escutelar posterior, ala anterior con 9-10 grupos de setas basales, vena marginal con 9-10 setas; terguito gástrico 7 con 6 setas y la tercera válvula 0.6 veces tan larga como el segundo valvífer.

Material examinado. Holotipo hembra: México, San Luis Potosí, Xilitla, Las Pozas, latitud N 2123', longitud W 98'59', 570 msnm, ex Aleyrodidae en pasto, 19.XI.2011, S. N. Myartseva, en laminilla. Paratipos: mismos datos que el holotipo, 4 hembras (S. N. Myartseva). El holotipo y dos paratipos están depositados en el ERMUCR, en Riverside, California, EU, y dos paratipos en el MIFA, en Cd. Victoria, Tamaulipas, México.

Etimología. El nombre de la especie se refiere a la localidad de colecta, Xilitla.

\section{Encarsia yumka Myartseva, sp. nov.}

Coloración. Cabeza amarilla, margen superior de la boca y clípeo negros. Ojos negro parduzco, ocelos rojizos. Antenas amarillo claro. Mesosoma amarillo, propodeo negro. Alas anteriores hialinas. Patas amarillo claro. Pecíolo negro. Gáster amarillo, terguitos 1 y 6 negros.

Estructura. Cabeza tan ancha como el mesosoma, 1.3 veces más ancha que alta. Frontovértice cerca de 0.5 veces la anchura de la cabeza. Ocelos situados en un triángulo obtuso pequeño. Distancia del ocelo posterior al margen del ojo cerca de 2 veces tan larga como la distancia entre los ocelos posteriores. Ojos cerca de 1.4 veces tan largos como las mejillas. Antenas (Fig. 5) insertadas cerca del margen bucal. Distancia entre los tórulos cerca de 0.6 veces tan larga como la distancia entre el tórulo y el ojo. Radícula antenal 2 veces tan larga como ancha. Escapo 4.3 veces tan largo como ancho. Pedicelo 1.4 veces tan largo como ancho. El primer segmento funicular es el más corto, 1.5-2 veces tan largo como ancho, segmentos 2 y 3 subiguales en longitud, cada uno 3 veces tan largo como ancho. Maza antenal de 3 segmentos, muy ligeramente más ancha y 1.25 veces más larga que el funículo. Segmentos flagelares 2-6 cada uno con una sensila. Lóbulo medio del mesoescudo con 4 pares de setas; cada axila con una seta, cada lóbulo lateral con 3 setas. Escutelo cerca de 0.6 veces tan largo como el lóbulo medio del mesoescudo y 2 veces tan ancho como largo. Sensilas escutelares placoideas cercanamente espaciadas. Par anterior de setas escutelares cerca de 2 veces más corta que el par posterior. Distancia entre las bases de las setas anteriores cerca de 0.5 veces tan larga como la distancia entre las bases de las setas posteriores. Ala anterior uniformemente setosa, 3.2 veces tan larga como ancha, su fleco marginal cerca de 0.4 veces la anchura alar máxima, base con 2 setas. Vena marginal (Fig. 6) ligeramente más larga que la vena submarginal y con 7 setas largas a lo largo del margen anterior. Ala posterior cerca de 10 veces tan larga como ancha, 

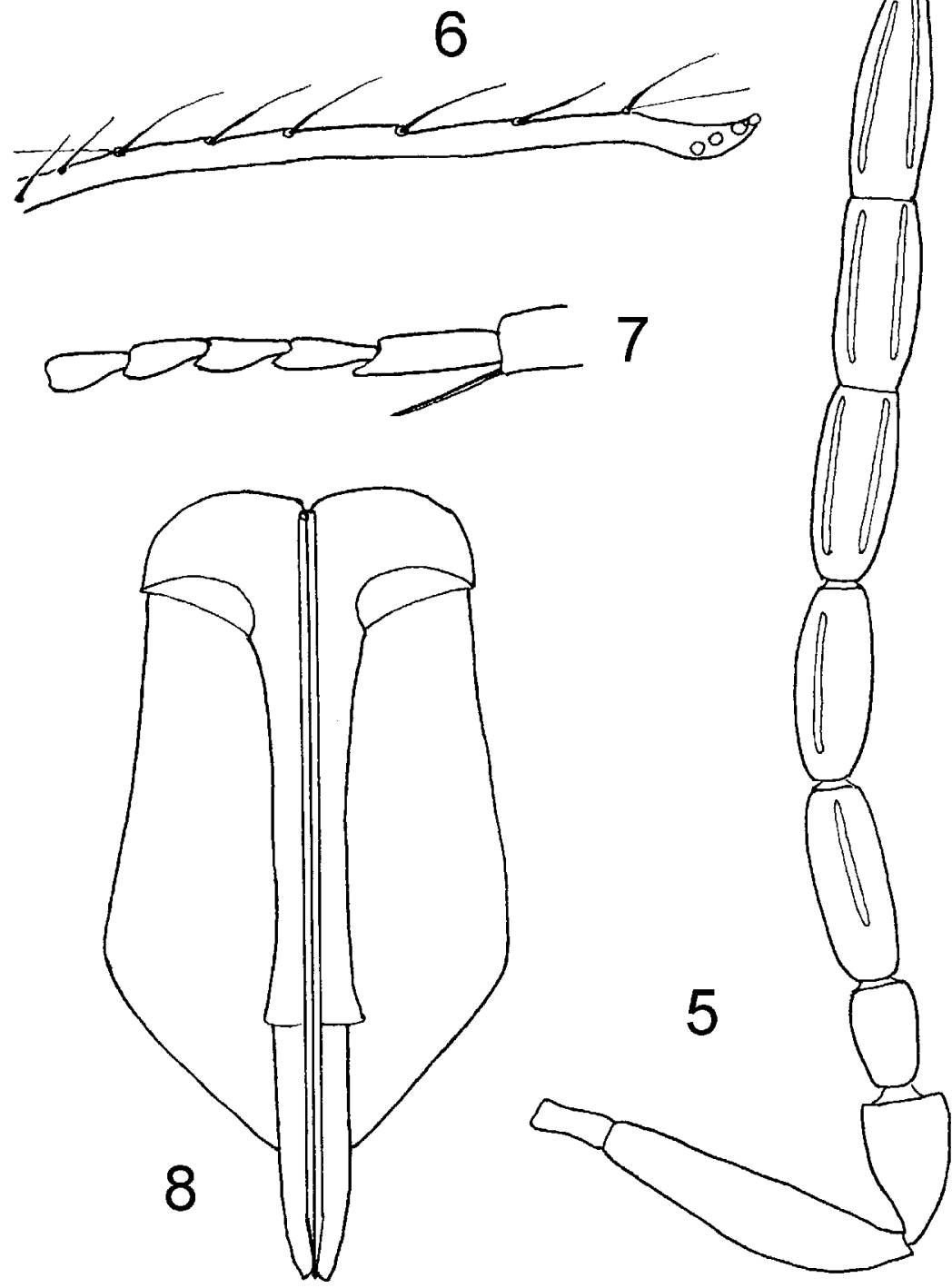

Figuras 5-8. Encarsia yumka Myartseva, sp. nov., hembra: 5 - antena, 6 - vena marginal del ala anterior, 7 - tarso de la pata media, 8 - ovipositor.

su fleco marginal cerca de 2 veces tan largo como la anchura alar máxima. Fórmula tarsal 5-5-5. Espuela de la tibia media más o menos tan larga como el basitarso (Fig. 7). Ovipositor (Fig. 8) muy ligeramente expuesto, cerca de 1.6 veces tan largo como la tibia media, tercera válvula cerca de 0.5 veces tan larga como el segundo valvífer. 
Longitud del cuerpo: $0.7 \mathrm{~mm}$.

Macho desconocido.

Comentarios. Encarsia yumka pertenece al grupo de especies citrella. Esta nueva especie es cercana a E. citrella (Howard), descrita de EU (Florida) (Howard, 1908). Se distinguen en lo siguiente: E. citrella presenta ala anterior ahumada debajo de la vena marginal, lóbulo medio del mesoescudo con 2 pares de setas, terguitos gástricos 3-5 pardo oscuro centralmente y la tercera válvula parduzca apicalmente. E. yumka tiene ala anterior hialina, lóbulo medio del mesoescudo con 4 pares de setas, terguitos gástricos 1 y 6 negros y la tercera válvula clara.

Material examinado. Holotipo hembra: México, Tabasco, Yumká, latitud N $17^{\circ}$ 59', longitud W 92 ${ }^{\circ}$ 58', ex Aleyrodidae en árbol desconocido, 7.XI.2009, S. N. Myartseva, en laminilla. Paratipos: mismos datos que el holotipo, 4 hembras (S. N. Myartseva). El holotipo y dos paratipos están depositados en el Entomological Research Museum de la Universidad de California-Riverside ERM-UCR), Estados Unidos, y dos paratipos en el Museo de Insectos de la FIC (MIFA), en Cd. Victoria, Tamaulipas, México.

Etimología. El nombre de la especie se refiere a la localidad de colecta, Yumká.

\section{DISCUSIÓN}

En la revisión de las especies de Encarsia de México (Myartseva \& Evans 2008) se registraron 88 especies para el país. Sin embargo, siguen describiéndose especies nuevas, como E. morela Myartseva del Estado de Morelos (Myartseva et al. 2010), E. tampicana Myartseva de Veracruz (Myartseva et al. 2011), y las descritas en el presente artículo. El género Encarsia Foerster es quizá el más diverso de Aphelinidae en México, por lo que nuevas especies y registros seguirán reportándose de manera continua.

Agradecimientos. Al proyecto PROMEP “Taxonomía y ecología de fauna y micobiota en comunidades forestales y cultivos” por su apoyo parcial para la realización de este trabajo y a la Universidad Autónoma de Tamaulipas, por el apoyo para efectuar este trabajo de campo, laboratorio y museo. A los revisores de este artículo, por sus atinados comentarios.

\section{LITERATURA CITADA}

De Santis, L. 1948. Estudio monográfico de los Afelínidos de la República Argentina (Hymenoptera, Chalcidoidea). Revista del Museo de La Plata, 5: 23-280.

Hayat, M. 1998. Aphelinidae of India (Hymenoptera: Chalcidoidea): a taxonomic revision. International Associated Publishers, Gainesville, USA.

Heraty, J., J. Woolley \& A. Polaszek. 2007. Catalog of the Encarsia of the World. http://www.faculty. ucr.edu/ heraty/Encarsia.cat.pdf. 87 pp. 
Howard, L. O. 1908. A key to the species of Prospaltella, with a table of hosts, and descriptions of four new species. Annals of the Entomological Society of America, 1: 281-284.

Huang, J. \& A. Polaszek. 1998. A revision of the Chinese species of Encarsia Foerster (Hymenoptera: Aphelinidae): parasitoids of whiteflies, scale insects and aphids (Hemiptera: Aphelinidae, Diaspididae, Aphidoidea). Journal of Natural History, 32: 1825-1966.

Myartseva, S. N. 2007. New species of Encarsia Förster from Veracruz, Mexico (Hymenoptera: Chalcidoidea: Aphelinidae). Zoosystematica Rossica, 16: 67-77.

Myartseva, S. N. 2009. Two new species of Encarsia (Hymenoptera: Aphelinidae), parasitoids of whiteflies (Homoptera: Aleyrodidae) from Mexico. Biosystematica, 3: 27-31.

Myartseva, S. N. \& G. A. Evans, 2008. Genus Encarsia Förster of Mexico (Hymenoptera: Chalcidoidea: Aphelinidae). A revisión, key and description of new species. Serie Avispas Parasíticas de Plagas y Otros Insectos, 3. Universidad Autónoma de Tamaulipas, Ciudad Victoria, México. 320 pp.

Myartseva, S. N. \& A. González-Hernández. 2008. Descripciones de dos especies nuevas de Encarsia (Hymenoptera: Aphelinidae) y nuevos registros de especies de este género para Chiapas y Jalisco, México. Acta Zoológica Mexicana, 24: 101-114.

Myartseva, S. N., E. Ruíz-Cancino \& J. M. Coronado-Blanco. 2008. Encarsia aurantii species-group (Hymenoptera: Aphelinidae), parasitoids of armored scales (Hemiptera: Diaspididae) in Mexico, with key and description of a new species. Zoosystematica Rossica, 7: 67-71.

Myartseva, S. N., G. Vejar-Cota \& E. Cortez-Mondaca. 2009. A new species of the genus Encarsia (Hymenoptera: Aphelinidae) - parasitoid of Aleurocybotus occiduus Russell (Hemiptera: Aleyrodidae) from Mexico. Russian Entomological Journal, 19: 123-126.

Myartseva, S. N., E. Ruíz-Cancino, J. M. Coronado-Blanco \& A. M. Corona-López. 2010. Especies de Encarsia (Hymenoptera: Aphelinidae) que parasitan Trialeurodes vaporariorum (Westwood) (Hemiptera: Aleyrodidae) en Tamaulipas y Morelos, México, y descripción de una especie nueva. Dugesiana, 17: 129-135.

Myartseva, S. N., E. Ruíz-Cancino \& J. M. Coronado-Blanco. 2011. Nueva especie de Encarsia Foerster y nuevos registros de avispas parasíticas (Chalcidoidea: Aphelinidae, Encyrtidae) de mosquitas blancas (Hemiptera: Aleyrodidae) de Veracruz, México. Acta Zoológica Mexicana (n. s.), 27: 803-809.

Nikolskaya, M. N. \& V. A. Yasnosh. 1966. Aphelinids of the European part of the USSR and the Caucasus (Hymenoptera, Aphelinidae). Opredeliteli po faune SSSR, 91. Moscow \& Leningrad, Nauka. 296 pp. (in Russian).

Noyes, J. S. 1982. Collecting and preserving chalcid wasps (Hymenoptera: Chalcidoidea). Journal of Natural History, 16: 315-334.

Noyes, J. S. 2011 (2003). Universal Chalcidoidea Database [online]. Worldwide Web electronic publication. www.nhm.ac.uk/entomology/chalcidoids/index.html. (Last updated: August 2011).

Polaszek, A. 1991. Egg parasitism in Aphelinidae (Hymenoptera: Chalcidoidea) with special reference to Centrodora and Encarsia species. Bulletin of Entomological Research, 81: 97-106.

Schmidt, S. \& A. Polaszek. 2007. The Australias species of Encarsia Förster (Hymenoptera, Chalcidoidea: Aphelinidae), parasitoides of whiteflies (Hemiptera, Sternorrhyncha, Aleyrodidae) and armoured scale insects (Hemiptera, Coccoidea: Diaspididae). Journal of Natural History, 41: 20992265.

Viggiani, G. 1987. New species of Encarsia Förster (Hymenoptera: Aphelinidae), parasitoids of whiteflies. Bollettino del Laboratorio di Entomologia Agraria "Filippo Silvestri" di Portici, 44: 33-41.

Woolley, J. B. 1997. Aphelinidae, pp. 134-150. In: Gibson, G.A.P., Huber, J.T. \& Woolley, J.B. (Eds.). Annotated keys to the genera of Nearctic Chalcidoidea (Hymenoptera). NRC Research Press, Ottawa, Canada. 794 pp. 